SECTION 31. Economic research, Finance, innovation.

Naumov Anatoly Aleksandrovich, Docent, candidate of Technical Sciences, Center of Applied Mathematical Research, Novosibirsk, Russia, e-mail: $\underline{\text { A_A_Naumov@mail.ru }}$

\title{
MODIFICATION OF NFV-CRITERIA BASED ON DETAILING OF FINANCIAL FLOWS OF PROJECTS METHOD
}

The paper discusses methods for evaluating the effectiveness of projects on the basis of compounding procedures for financial flows. In particular, new schemes for calculating of NFV-index constructed on the basis of detailed flows are proposed.

Key words: Investment projects, NFV criteria, method of detailed flows.

\section{УДК 330.46: 658.155}

\section{МОДИФИКАЦИЯ КРИТЕРИЯ NFV НА ОСНОВЕ МЕТОДА ДЕТАЛИЗАЦИИ ФИНАНСОВЫХ ПОТОКОВ ПРОЕКТОВ}

B работе рассмотрены методы оценивания эффективности проектов на основе процедуры компаундирования финансовых потоков. $B$ частности, предложены новые схемы расчета показателя NFV, которые построены на основе метода детализаџии потоков.

Ключевые слова: инвестиционные проекты, NFV критерий, метод детализации потоков.

Такой показатель назван в работе как $\mathrm{NFV}_{\mathrm{DF}}$ (Net Future Value for Disintegration Flows). Детализация потоков позволяет приблизить задачу анализа инвестиционных проектов к практическому использованию.

Постановка задачи. Пусть для некоторого инвестиционного проекта известны входной и выходной потоки в виде: $\mathrm{F}_{\text {in }}(\mathrm{t}), \mathrm{t}=\mathrm{t}_{0}, \mathrm{t}_{1}, \mathrm{t}_{2}, \ldots,\left(\mathrm{t}_{\mathrm{m}}=\right.$ $\mathrm{T})$, - входной финансовый поток, $\mathrm{F}_{\text {out }}(\mathrm{t}), \mathrm{t}=\mathrm{t}_{0}, \mathrm{t}_{1}, \mathrm{t}_{2}, \ldots,\left(\mathrm{t}_{\mathrm{m}}=\mathrm{T}\right)$, выходной финансовый поток. Для простоты записи формул расчета показателей, будем предполагать, что моменты времени $\mathrm{t}_{0}, \mathrm{t}_{1}, \mathrm{t}_{2}, \ldots,\left(\mathrm{t}_{\mathrm{m}}=\right.$ T) - положительные целые числа и все интервалы между соседними отсчетами равны единице времени. Кроме этого полагаем, что все ставки согласованы с этой единицей времени. Если обозначить через $r_{\text {in }}$ ставку заимствования инвестируемых финансовых средств, а через $r_{\text {out }}$ - ставку внешнего использования выходного финансового потока, то одна из простейших схем расчета показателя NFV (Net Future Value) выглядит следующим образом:

$$
\operatorname{NFV}(T)=F_{\text {out }}^{\Sigma}(T)-F_{\text {in }}^{\Sigma}(T) \text {; }
$$




$$
\begin{aligned}
& \mathrm{F}_{\text {out }}^{\Sigma}(\mathrm{T})=\sum_{\mathrm{t}=\mathrm{t}_{0}}^{\mathrm{t}=\mathrm{t}_{\mathrm{m}}} \mathrm{F}_{\text {out }}(\mathrm{t}) \cdot\left(1+\mathrm{r}_{\text {out }}\right)^{\mathrm{T}-\mathrm{t}} \\
& \mathrm{F}_{\text {in }}^{\Sigma}(\mathrm{T})=\sum_{\mathrm{t}=\mathrm{t}_{0}}^{\mathrm{t}=\mathrm{t}_{\mathrm{m}}} \mathrm{F}_{\text {in }}(\mathrm{t}) \cdot\left(1+\mathrm{r}_{\mathrm{in}}\right)^{\mathrm{T}-\mathrm{t}} .
\end{aligned}
$$

Заметим, что приведенный доход оценивается на момент времени $\mathrm{T}$. Тогда доходность можно оценить по формулам:

$$
\operatorname{IRR}=\left\{\mathrm{r} \mid \sum_{\mathrm{t}} \mathrm{F}_{\mathrm{in}}(\mathrm{t}) \cdot(1+\mathrm{r})^{\mathrm{T}-\mathrm{t}}=\mathrm{F}_{\text {out }}^{\Sigma}(\mathrm{T})\right\}
$$

или

$$
\operatorname{IRR}=\left\{\mathrm{r} \mid \sum_{\mathrm{t}} \mathrm{F}_{\mathrm{in}}(\mathrm{t}) \cdot(1+\mathrm{r})^{\mathrm{T}-\mathrm{t}}=\mathrm{NFV}(\mathrm{T})\right\} .
$$

Здесь для простоты записи вместо обозначения суммы в виде $\sum_{\mathrm{t}=\mathrm{t}_{0}}^{\mathrm{t}=\mathrm{t}_{\mathrm{m}}} \ldots$ использовано более компактное обозначение $-\sum_{\mathrm{t}} \ldots$. Использование одной из этих формул зависит от того, хотим ли мы оценить доходность проекта на основании наращенного (компаундированного) выходного потока или общего дохода. Следует заметить, что даже для эффективного проекта доходность, оцененная в соответствии с формулой (3), может принимать отрицательные значения. Это объясняется тем обстоятельством, что общий доход NFV(T) может составлять лишь часть общих затрат (инвестиций) в проект $\sum_{\mathrm{t}} \mathrm{F}_{\mathrm{in}}(\mathrm{t})$.

Принцип детализации. Проведем детализацию входного и выходного потоков проекта. Будем анализировать последовательно элементы множества входного потока $\left\{\mathrm{F}_{\text {in }}(\mathrm{t})\right\}, \mathrm{t}=\mathrm{t}_{0}, \mathrm{t}_{1}, \mathrm{t}_{2}, \ldots, \mathrm{t}_{\mathrm{m}}$, для возрастающих значений моментов времени. Пусть $\mathrm{F}_{\text {in }}\left(\mathrm{t}_{0}\right)$ - это заемные средства под ставку $\mathrm{r}_{\text {in,0 }}$, которые необходимо будет погашать в виде одинаковых выплат в интервале времени $\left[\mathrm{t}_{0} ; \mathrm{T}_{0}\right]$. Хорошо известно, что в этом случае величины кредита и погашений кредита связаны между собой следующей формальной записью:

$$
\mathrm{F}_{\text {in }}\left(\mathrm{t}=\mathrm{t}_{0}\right)=\mathrm{F}_{\text {in }}\left(\mathrm{t}_{0}\right)=\mathrm{F}_{\mathrm{in}, \mathrm{RC}, 0} \cdot \frac{1-\left(1+\mathrm{r}_{\mathrm{in}, 0}\right)^{-\mathrm{n}_{0}}}{\mathrm{r}_{\mathrm{in}, 0}} .
$$

Здесь $\mathrm{F}_{\mathrm{in}, \mathrm{RC}, 0}(R C$ - Repayment of Credit $)$ - выплаты за кредит в размере $\mathrm{F}_{\mathrm{in}}\left(\mathrm{t}_{0}\right), \mathrm{n}_{0}$ - количество временных тактов в интервале времени $\left[\mathrm{t}_{0} ; \mathrm{T}_{0}\right]$, в которые происходят выплаты по кредиту, для целых $\mathrm{t}_{0}$ и $\mathrm{T}_{0}$ будет выполняться: $\mathrm{n}_{0}=\mathrm{T}_{0}-\mathrm{t}_{0}$ (при условии, что кредит выделяется в момент времени $t_{0}$, a погашение его происходит в конце каждого интервала времени $\left[\mathrm{t}_{\mathrm{i}} ; \mathrm{t}_{\mathrm{i}+1}\right], \mathrm{i}=0,1,2, \ldots,\left(\mathrm{n}_{0}-1\right), \mathrm{t}_{\mathrm{n}_{0}}=\mathrm{T}_{0}$.

Из формулы (4) получим значение выплат по кредиту:

$\mathrm{F}_{\mathrm{in}, \mathrm{RC}, 0}=\mathrm{F}_{\mathrm{in}}\left(\mathrm{t}_{0}\right) \cdot \frac{\mathrm{r}_{\mathrm{in}, 0}}{1-\left(1+\mathrm{r}_{\mathrm{in}, 0}\right)^{-\mathrm{n} 0}}$.

Таким образом, элемент входного потока $\mathrm{F}_{\mathrm{in}}\left(\mathrm{t}_{0}\right)$ породил поток выплат по кредиту: $\mathrm{F}_{\mathrm{in}, \mathrm{DF}, 0}=\left(0, \mathrm{~F}_{\mathrm{in}, \mathrm{RC}, 0}, \mathrm{~F}_{\mathrm{in}, \mathrm{RC}, 0}, \ldots \mathrm{F}_{\mathrm{in}, \mathrm{RC}, 0}\right)-$ вектор из $\left(\mathrm{n}_{0}+1\right)$-го элемента, каждый из элементов вектора привязан к моментам времени $\mathrm{t}_{0}, \mathrm{t}_{1}, \mathrm{t}_{2}, \ldots, \mathrm{t}_{\mathrm{n}_{0}}=\mathrm{T}_{0}$ соответственно. 
Переходим к следующему моменту времени $t=t_{1}$ и элементу входного потока $\mathrm{F}_{\mathrm{in}}\left(\mathrm{t}_{1}\right)$. Предположим, что эти вложения тоже покрываются кредитом со ставкой $r_{\text {in,1 }}$ и временным интервалом его погашения $\left[\mathrm{t}_{1} ; \mathrm{T}_{1}\right]$, причем для целых $\mathrm{t}_{1}$ и $\mathrm{T}_{1}$ количество выплат по второму кредиту равно $\mathrm{n}_{1}=\mathrm{T}_{1}-\mathrm{t}_{1}$. Тогда элемент входного потока $\mathrm{F}_{\mathrm{in}}\left(\mathrm{t}_{1}\right)$ в результате его детализации приведет к образованию вектора выплат вида: $\mathrm{F}_{\mathrm{in}, \mathrm{DF}, 1}=\left(0, \mathrm{~F}_{\mathrm{in}, \mathrm{RC}, 1}, \mathrm{~F}_{\mathrm{in}, \mathrm{RC}, 1}, \ldots \mathrm{F}_{\mathrm{in}, \mathrm{RC}, 1}\right)$, элементы которого относятся к моментам времени $\mathrm{t}_{1}, \mathrm{t}_{2}, \ldots, \mathrm{t}_{\mathrm{n}_{1}}=\mathrm{T}_{1}$ соответственно. В этом векторе элементы равны:

$\mathrm{F}_{\mathrm{in}, \mathrm{RC}, 1}=\mathrm{F}_{\mathrm{in}}\left(\mathrm{t}_{1}\right) \cdot \frac{\mathrm{r}_{\mathrm{in}, 1}}{1-\left(1+\mathrm{r}_{\mathrm{in}, 1}\right)^{-\mathrm{n}_{1}}}$.

Допустим, что для момента времени $\mathrm{t}=\mathrm{t}_{2}$ элемент потока $\mathrm{F}_{\mathrm{in}}\left(\mathrm{t}_{2}\right)$ будет обеспечен собственными средствами. Тогда соответствующий этому элементу вектор $\mathrm{F}_{\mathrm{in}, \mathrm{RC}, 2}^{\Sigma}$ будет иметь только одну компоненту, привязанную к моменту времени $\mathrm{t}_{2}: \mathrm{F}_{\mathrm{in}, \mathrm{DF}, 2}=\left(\mathrm{F}_{\text {in }}\left(\mathrm{t}_{2}\right)\right)$.

И так продолжаем применять эту процедуру детализации элементов входного потока до момента времени $\mathrm{t}=\mathrm{t}_{\mathrm{m}}$ (при этом получим вектор $\left.\mathrm{F}_{\mathrm{in}, \mathrm{DF}, \mathrm{m}}\right)$. Затем сводим все векторы $\mathrm{F}_{\mathrm{in}, \mathrm{DF}, \mathrm{i}} \mathrm{i}=0,1,2, \ldots, \mathrm{m}$, в один общий вектор с учетом привязки компонент этих векторов к моментам времени и, суммируя значения векторов, относящихся к одним моментам времени.

Итак, в результате свертки векторов $\mathrm{F}_{\mathrm{in}, \mathrm{DF}, \mathrm{i}} \mathrm{i}=0,1,2, \ldots, \mathrm{m}$, получим новый вектор $\mathrm{F}_{\mathrm{in}, \mathrm{DF}}^{\Sigma}$. Условно свертку векторов обозначим следующим образом: $\quad \mathrm{F}_{\mathrm{in}, \mathrm{DF}}^{\Sigma}=\oplus_{\mathrm{i}=0}^{\mathrm{m}} \mathrm{F}_{\mathrm{in}, \mathrm{DF}, \mathrm{i}}$. Для определенности положим, что компоненты вектора $\mathrm{F}_{\mathrm{in}, \mathrm{DF}}^{\Sigma}$ относятся $\mathrm{K}$ моментам времени $\mathrm{t}=\mathrm{t}_{0}, \mathrm{t}_{1}, \mathrm{t}_{2}, \ldots, \mathrm{t}_{\mathrm{DF}}=\mathrm{T}_{\mathrm{DF}}$ и пусть выполняется неравенство $\mathrm{T}_{\mathrm{DF}} \geq \mathrm{T}$.

Перейдем к детализации элементов выходного потока $\mathrm{F}_{\text {out }}(\mathrm{t}), \mathrm{t}=$ $\mathrm{t}_{0}, \mathrm{t}_{1}, \mathrm{t}_{2}, \ldots, \mathrm{t}_{\mathrm{m}}$. Основная идея процедуры детализации выходного потока состоит в том, чтобы расписать (распределить) элементы этого потока в два потока: один будет показывать, как компенсируются (погашаются) элементы потока $\mathrm{F}_{\mathrm{in,DF}}^{\Sigma}$ (будем обозначать его через $\mathrm{F}_{\text {out,IP }}^{\Sigma}$, где IP - Internal Projects, внутренние проекты) и второй - поток, который может быть выведен из данного проекта (процесса) и использован в других проектах

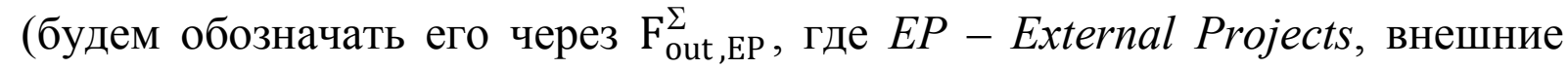
проекты). Следует иметь в виду, что наряду с непосредственно элементами этих двух потоков $\left(\mathrm{F}_{\text {out }, \text { IP }}^{\Sigma}\right.$ и $\left.\mathrm{F}_{\text {out,EP }}^{\Sigma}\right)$ в результате применения процедуры детализации будут получены два соответствующих им вектора параметров $-\pi_{\text {out ,IP }}^{\Sigma} \pi_{\text {out ,EP }}^{\Sigma}$ Эти параметры представляют из себя ставки, по которым элементы $\mathrm{F}_{\text {out,IP }}^{\Sigma}$ ожидают их использования в качестве погашений потока $\mathrm{F}_{\text {in,DF }}^{\Sigma}$ (это параметры $\pi_{\text {out,IP }}^{\Sigma}$ ), а элементы $\mathrm{F}_{\text {out,EP }}^{\Sigma}-$ используются во внешних проектах (за это отвечают параметры $\pi_{\text {out,EP }}^{\Sigma}$ ). Строго говоря, 
аналогичные векторы параметров сопровождают и компоненты вектора $\mathrm{F}_{\mathrm{in}, \mathrm{DF}}^{\Sigma}$ (и векторов $\mathrm{F}_{\mathrm{in}, \mathrm{DF}, \mathrm{i}} \mathrm{i}=0,1,2, \ldots, \mathrm{m}$ ). Для этих векторов параметры характеризуют элементы входного потока: ставки заимствования $-\mathrm{r}_{\mathrm{in,i}}$, $\mathrm{i}=0,1,2, \ldots, \mathrm{m}$; количество временных тактов погашения кредитов $-\mathrm{n}_{\mathrm{i}}$, $\mathrm{i}=0,1,2, \ldots, \mathrm{m}$. Как и для элементов входного потока, в данном случае можно записать равенства: $\mathrm{F}_{\text {out }, \mathrm{IP}}^{\Sigma}=\oplus_{\mathrm{i}=0}^{\mathrm{m}} \mathrm{F}_{\text {out }, \mathrm{IP}, \mathrm{i}}$ и $\mathrm{F}_{\text {out }}=\mathrm{F}_{\text {out }, \mathrm{IP}}^{\Sigma} \oplus \mathrm{F}_{\text {out }, \mathrm{EP}}^{\Sigma}$. Здесь $\mathrm{F}_{\text {out }}=\left(\mathrm{F}_{\text {out }}\left(\mathrm{t}_{0}\right), \mathrm{F}_{\text {out }}\left(\mathrm{t}_{1}\right), \ldots, \mathrm{F}_{\text {out }}\left(\mathrm{t}_{\mathrm{m}}\right)\right)$.

Очевидно, что элементы вектора $\mathrm{F}_{\text {out,IP }}^{\Sigma}$ должны полностью покрывать (компенсировать) компоненты вектора $\mathrm{F}_{\mathrm{in}, \mathrm{DF}}^{\Sigma}$ для всех моментов времени $\mathrm{t}=\mathrm{t}_{0}, \mathrm{t}_{1}, \mathrm{t}_{2}, \ldots, \mathrm{t}_{\mathrm{m}}$. Формально это можно записать таким образом: $\mathrm{F}_{\text {out }, I P}^{\Sigma}(\mathrm{t})=\mathrm{F}_{\mathrm{in}, \mathrm{DF}}^{\Sigma}(\mathrm{t})$ для всех $\mathrm{t} \in\left\{\mathrm{t}_{0}, \mathrm{t}_{1}, \mathrm{t}_{2}, \ldots, \mathrm{t}_{\mathrm{m}}\right\}$. Если это условие не выполняется, то необходимо повторить процедуру детализации сначала (поменяв источники кредитования, условия кредитования и т.д.) или сделать вывод о том, что проект не является эффективным. Если равенства $\mathrm{F}_{\text {out }, \mathrm{IP}}^{\Sigma}(\mathrm{t})=\mathrm{F}_{\mathrm{in}, \mathrm{DF}}^{\Sigma}(\mathrm{t})$ для всех $\mathrm{t} \in\left\{\mathrm{t}_{0}, \mathrm{t}_{1}, \mathrm{t}_{2}, \ldots, \mathrm{t}_{\mathrm{m}}\right\}$ выполнились, то можно перейти к оцениванию показателя эффективности $\mathrm{NFV}_{\mathrm{DF}}$ в соответствии $\mathrm{c}$ формулой:

$$
\mathrm{NFV}_{\mathrm{DF}}=\sum_{\mathrm{t}} \mathrm{F}_{\text {out }, E P}^{\Sigma}(\mathrm{t}) \cdot\left(1+\mathrm{r}_{\text {out }, E P}(\mathrm{t})\right)^{\mathrm{T}-\mathrm{t}} .
$$

Здесь $\mathrm{F}_{\text {out,EP }}^{\Sigma}(\mathrm{t})$ - элемент вектора $\mathrm{F}_{\text {out,EP }}^{\Sigma}$, относящийся к моменту времени $t, r_{\text {out,EP }}(t)$ - ставка внешнего использования (во внешних проектах) средств в размере $\mathrm{F}_{\text {out,EP }}^{\Sigma}(\mathrm{t})$.

Отметим положительные моменты, связанные с показателем $\mathrm{NFV}_{\mathrm{DF}}$.

Первое. Все ставки, используемые в расчетах показателя, имеют ясный смысл и выбираются достаточно однозначно (это либо банковские ставки для кредитов, либо ставки по депозитам, либо ставки внешних проектов, эффективность которых оценена и т.д.).

Второе. Значение показателя интерпретируется достаточно просто это доход от проекта, который будет получен на момент времени $\mathrm{t}=\mathrm{T}$.

Третье. Фактически элементы $\mathrm{F}_{\text {out,EP }}^{\Sigma}(\mathrm{t})$ в формуле для показателя (7) равны разностям между компонентами детализированных векторов выходного и входного потоков.

Опираясь на выражение (7) для оценивания дохода $\mathrm{NFV}_{\mathrm{DF}}$, можно предложить расчетные схемы оценивания доходности проекта:

$$
\begin{aligned}
& I_{R R_{D F}, \mathrm{in}, \mathrm{NFV}+\mathrm{DF}}=\left\{\mathrm{r} \mid \sum_{\mathrm{t}} \mathrm{F}_{\mathrm{in}}(\mathrm{t}) \cdot(1+\mathrm{r})^{\mathrm{T}-\mathrm{t}}=\mathrm{NFV}_{\mathrm{DF}}+\sum_{\mathrm{t}} \mathrm{F}_{\mathrm{in}, \mathrm{DF}}^{\mathrm{L}}(\mathrm{t})\right\}, \\
& \mathrm{IRR}_{\mathrm{DF}, \mathrm{in}}=\left\{\mathrm{r} \mid \sum_{\mathrm{t}} \mathrm{F}_{\mathrm{in}}(\mathrm{t}) \cdot(1+\mathrm{r})^{\mathrm{T}-\mathrm{t}}=\mathrm{NFV}_{\mathrm{DF}}\right\}, \\
& \mathrm{IRR}_{\mathrm{DF}, \mathrm{DF}}=\left\{\mathrm{r} \mid \sum_{\mathrm{t}} \mathrm{F}_{\mathrm{in}, \mathrm{DF}}^{\Sigma}(\mathrm{t}) \cdot(1+\mathrm{r})^{\mathrm{T}-\mathrm{t}}=\mathrm{NFV}_{\mathrm{DF}}\right\} .
\end{aligned}
$$
всех трех случаях расчета доходности дается ответ на вопрос: каково влияние потоков $\left(\left(F_{i n}(t)+F_{i n, D F}^{\Sigma}(t)\right), F_{i n}(t)\right.$ или $\left.F_{i n, D F}^{\Sigma}(t)\right)$ на общий доход проекта $\left(\mathrm{NFV}_{\mathrm{DF}}\right)$. 
Для нахождения «глобального» срока окупаемости проекта, т.е. такого момента времени, правее которого на оси времени поток $\mathrm{F}_{\text {out,EP }}^{\Sigma}$ Hе обращается в ноль, можно воспользоваться формулой:

$$
\mathrm{PP}=\left\{\min \mathrm{t}^{*} \in\left\{\mathrm{t}_{0}, \mathrm{t}_{1}, \mathrm{t}_{2}, \ldots, \mathrm{t}_{\mathrm{m}}=\mathrm{T}\right\} \mid \begin{array}{c}
\mathrm{F}_{\text {out }, \mathrm{EP}}(\mathrm{t})>0, \\
\forall_{\mathrm{t}} \mathrm{t} \in\left\{\mathrm{t}^{*}, \mathrm{t}^{*}+1, \ldots, \mathrm{t}_{\mathrm{m}}\right\}
\end{array}\right\} .
$$

\section{Литература}

1. Наумов А. А. Теоретические и прикладные вопросы моделирования бизнес-процессов. Модели, алгоритмы, программы: Монография/ А.А. Наумов. - LAP LAMBERT Academic Publishing, 2012. - 464 c. [https://www.ljubljuknigi.ru/store/ru/book/Tеоретические-и-прикладныевопросы-моделирования-бизнес-процессов/isbn/978-3-8383-6534-3]

2. Список трудов [Электронный ресурc]. URL: https://sites.google.com/site/ anatolynaumov2011/home/spisok-trudov-list-of-papers (дата обращения: 25.09.2013). 